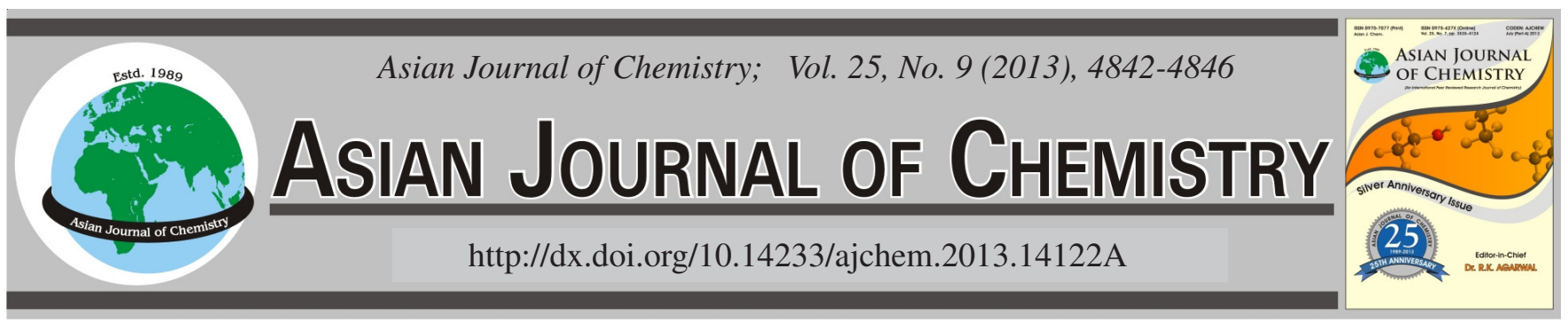

\title{
Characterization and Evaluation of Semi-IPN Hydrogels Consisted of Pectin and Poly(acrylonitrile) for Drug Delivery Systems
}

\section{Mohammad Sadeghi", Anahita Gudarzi, Soudeh Safari, Hadis Shahsavari and Hossein Sadeghi}

Department of Chemistry, Science Faculty, Islamic Azad University, Arak Branch, Arak, Iran

*Corresponding author: E-mail: m-sadeghi@iau-arak.ac.ir

\begin{abstract}
In the present paper, attention is paid to synthesis and evolution swelling behaviour of a superabsorbent hydrogel based on pectin (Pec) and polyacrylonitrile (PAN) carriers for the controlled delivery of ephedrine. The physical mixture of pectin and polyacrylonitrile was hydrolyzed by $\mathrm{NaOH}$ solution to yield pectin-poly(sodium acrylate-co-acrylamide) superabsorbent hydrogel. A proposed mechanism for hydrogel formation was suggested and the structure of the product was established using FTIR and SEM spectroscopies, respectively. The pectin-poly(sodium acrylate-co-acrylamide) hydrogel exhibited a pH-responsiveness character so that a swelling-deswelling pulsatile behaviour was recorded at pHs 1.6 and 7.4. In addition, the delivery behaviour of ephedrine from the hydrogel bead was studied. The amount of ephedrine released from the hydrogel bead at $\mathrm{pH} 1.6$ was relatively low $(15 \%)$, while this value approached $87 \%$ at $\mathrm{pH} 7.4$. The results clearly suggested that the pectin-bsaed hydrogel may be a potential polymeric carrier for drug delivery in the intestinal tract.
\end{abstract}

Key Words: Pectin, Polyacrylonitrile, Superabsorbent, Drug delivery systems, Ephedrine.

\section{INTRODUCTION}

Hydrogels, chemically or physically crosslinked threedimensional networks composed of hydrophilic polymers, can absorb and retain large amounts of aqueous fluids and the absorbed water is hardly removable even under some pressure. Due to excellent properties compared with traditional water absorbing materials, hydrogels have drawn much attention in a wide variety of fields such as drug delivery system, wound dressings, gel actuators, artificial organs, medical pharmaceuticals and contact lenses ${ }^{1-3} \cdot \mathrm{pH}$-sensitive hydrogels have attracted increasing attention due to their unique properties. Swelling of such hydrogels in the stomach is minimal and thus the drug release is also minimal. Due to increase in $\mathrm{pH}$, the swelling degree increases as the hydrogels pass down the intestinal tract. A variety of synthetic or natural polymers with acidic or basic pendant groups have been employed to fabricate $\mathrm{pH}$-sensitive hydrogels for getting the desired controlled release of drugs ${ }^{4}$. The use of natural polymers in the design of $\mathrm{pH}$ sensitive hydrogels has received much attention due to their excellent biocompatibility. The aim of this work was not only to characterize new type of superabsorbents, but also to evaluate the usefulness and feasibility of these polysaccharidebased superabsorbents for orally administered drug delivery system (DDS).

\section{EXPERIMENTAL}

Pectin (chemical grade, MW 50000) was purchased from Merck Chemical Co. (Germany). Polyacrylonitrile (PAN) was synthesized through a method mentioned in the literature ${ }^{5}$. The drug, ephedrine, was obtained from Jaberebne Hayan Pharmaceutical Co. (Tehran, Iran). The chemical structure of ephedrine is shown in Fig. 1. All other chemicals were analytical grade and used without further purification double distilled water was used for the hydrogel preparation and swelling measurements.

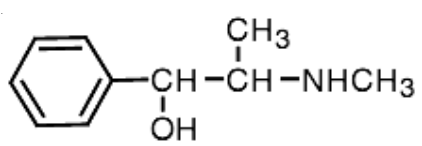

Fig. 1. Chemical structure of ephedrine

Hydrogel preparation: A facial one step preparative method was used for synthesis of Pec-poly(sodium acrylateco-acrylamide) hydrogel, Pec-poly(NaAA-co-AAm), hydrogel. A general procedure for alkaline hydrolysis of Pec -PAN mixture was conducted as follows. Pectin (0.50-1.33 g) was added to a three-neck reactor equipped with a mechanical stirrer (Heidolph RZR 2021, three blade propeller type, 50$500 \mathrm{rpm}$ ), including $35 \mathrm{~mL}$ doubly distilled water. The reactor 
was immersed in a thermostated water bath. After complete dissolution of pectin to form a homogeneous solution, certain amount of sodium hydroxide $(0 / 25-2 / 5 \mathrm{~N})$ was added to the pectin solution at desired temperature (alkalization temperature, $20-120^{\circ} \mathrm{C}$ ). The mixture was allowed to stir for certain times (alkalization times, 20-90 min). The various amount of polyacrylonitrile (0.50-4.50 g) was dispersed in the reaction mixture to saponify for certain times and temperatures (alkaline time and temperature). During the saponification $\mathrm{NH}_{3}$ gas was evolved and a change in colour is observed from red to light yellow. This change of colour was an indication of the reaction completion. The pasty mixture was allowed to cool to room temperature and neutralized to $\mathrm{pH} 8$ by addition of $10 \mathrm{wt} \%$ aqueous acetic acid solution. Then the gelled product was scissored to small pieces and poured in ethanol $(200 \mathrm{~mL})$ to dewater for $5 \mathrm{~h}$. The hardened particles were filtered and dried in oven $\left(50^{\circ} \mathrm{C}, 10\right.$ h). After grinding, the powdered superabsorbent hydrogel was stored away from moisture, heat and light.

Studies of the dynamic and equilibrium degree of swelling: The tea bag (i.e., a 100 mesh nylon screen) containing an accurately weighed powdered sample $(0.5 \pm 0.001 \mathrm{~g})$ with average particle sizes between 40-60 mesh (250-350 $\mu \mathrm{m})$ was immersed entirely in distilled water $(200 \mathrm{~mL})$ or desired salt solution $(100 \mathrm{~mL})$ and allowed to soak for $3 \mathrm{~h}$ at room temperature. The tea bag was hung up for $15 \mathrm{~min}$ in order to remove the excess fluid. The equilibrated swelling (ES) was measured twice using the following equation:

$$
\mathrm{ES}(\mathrm{g} / \mathrm{g})=\frac{\text { Weight of swollen gel }- \text { Weight of dried gel }}{\text { Weight of dried gel }}
$$

The accuracy of the measurements was $\pm 3 \%$.

pH-Sensitivity: pH-Sensitivity of the hydrogel was investigated in terms of swelling and deswelling of the final product at two basic ( $\mathrm{pH} 7.4)$ and acidic $(\mathrm{pH}$ 1.6) solutions, respectively. Swelling capacity of the hydrogels at each $\mathrm{pH}$ was measured according to eqn. 1 at consecutive time intervals (0.5 h).

Standard absorbance curve: The standard calibration curve of the absorbance as a function of drug concentration was studied at $276 \mathrm{~nm}$ on the UV spectrophotometer.

Instrumental analysis: Fourier transform infrared (FTIR) spectra of samples were taken in $\mathrm{KBr}$ pellets, using an $\mathrm{ABB}$ Bomem MB-100 FTIR spectrophotometer (Quebec, Canada), at room temperature. The surface morphology of the gel was examined using scanning electron microscopy (SEM). After Soxhlet extraction with methanol for $24 \mathrm{~h}$ and drying in an oven, superabsorbent powder was coated with a thin layer of gold and imaged in a SEM instrument (Leo, 1455 VP).

Drug loading efficiency and in vitro drug release: Powdered samples $(1 \pm 0.0001 \mathrm{~g})$, with average particle sizes between 40 and 60 mesh (250-420 mm), were accurately weighted and immersed in an alkaline solution of ephedrine ( $0.54 \mathrm{~g}$ dissolved in $50 \mathrm{~mL}$ distilled water) at $37{ }^{\circ} \mathrm{C}$ for $25 \mathrm{~h}$. The swolen hydrogels loaded with drug were placed in a vacuum oven and dried under vacuum at $37{ }^{\circ} \mathrm{C}$. The loading amount of drug in the hydrogels was calculated from the decrease in the concentration of the ephedrine solution which was determined using a UV spectrophotometer (UV-1201,
Shimadzu, Kyoto, Japan). The loading efficiency of the pectinbased hydrogels was calculated as the ratio of the final to the initial ephedrine concentration ${ }^{6-8}$.

In vitro release was carried out in duplicate by incubating $0.01 \pm 0.0001 \mathrm{~g}$ of the ephedrine -loaded hydrogels into a cellophane membrane dialy sis bag (DSIGM A-ALD RICH) in $50 \mathrm{~mL}$ of buffer solution (either $\mathrm{pH} 1.6$ or 7.4 ) at $37^{\circ} \mathrm{C}$. At specific time intervals, $1 \mathrm{~mL}$ aliquots of sample was withdrawn and after suitable dilution the concentration of drug released was measured by UV spectrophotometer. The drug release per cent was calculated twice using the following equation:

$$
\text { Released drug }(\%)=\frac{\mathrm{R}_{\mathrm{t}}}{\mathrm{L}} \times 100
$$

where $L$ and $R_{t}$ represent the initial amount of drug loaded and the final amount of drug released at time $t$.

\section{RESULTS AND DISCUSSION}

Mechanism of hydrogel formation and IR analysis: A general reaction mechanism for Pec-poly(AN) hydrogel formation is shown in Scheme-I. At the first step, hydroxyl groups of pectin substrate was converted to corresponding alkoxide ions using sodium hydroxide solution (Scheme-I). Then, these macroalkoxides initiate crosslinking reaction between some adjacent polyacrylonitrile pendant chains. This reaction leads to intermediate formation of naphthyridine cyclic structures (including imine, $-\mathrm{C}=\mathrm{N}-$, conjugated bonds) with deep red colour. The intermediate was then hydrolyzed using residual sodium hydroxide aqueous solution to produce hydrophilic carboxamide and carboxylate groups (Scheme-I) with a resulting colour change from red to light yellow. This sharp colour change was used as an indication to halt the alkaline treatment. However, incomplete hydrolyzed structures may also give rise to a few crosslinking points result in a loosely crosslinked network. It has been reported, in the case of H-SPAN, a maximum conversion of $70 \%$ of nitrile to carboxyl groups and the remaining $30 \%$ are amide groups ${ }^{9}$. In fact, details of the chemical processes and mechanism involved in H-SPAN synthesis are not yet well understood. For instance, the incomplete hydrolysis is interpreted as being related to steric and polar factors ${ }^{10}$. Oh et al. ${ }^{11}$ suggested that condensation might also occur between carboxyl and amide groups to form imide structures. Therefore, in the case of our hydrogel, Pec-poly(NaAA-co-AAm), we realized that precise control of the ratio is practically impossible.

Infrared spectroscopy was carried out to confirm the chemical structure of the hydrogel. Fig. 2 shows the FTIR spectra of Pec-PAN physical mixture and the resulted hydrogel, Pec-poly(NaAA-co-AAm). The band observed at $2242 \mathrm{~cm}^{-1}$ can be attributed to stretching of - $\mathrm{CN}$ group of polyacrylonitrile (Fig. 2b). The hydrogel comprise an pectin backbone with side chains that carry carboxamide and carboxylate functional groups that are evidenced by three new peaks at 1407, 1556 and $1675 \mathrm{~cm}^{-1}$ (Fig. 2c). These peaks attributed to $\mathrm{C}=\mathrm{O}$ stretching in carboxamide functional groups and symmetric and asymmetric stretching modes of carboxylate groups, respectively ${ }^{12}$. The stretching band of - $\mathrm{NH}$ overlapped with the $-\mathrm{OH}$ stretching 


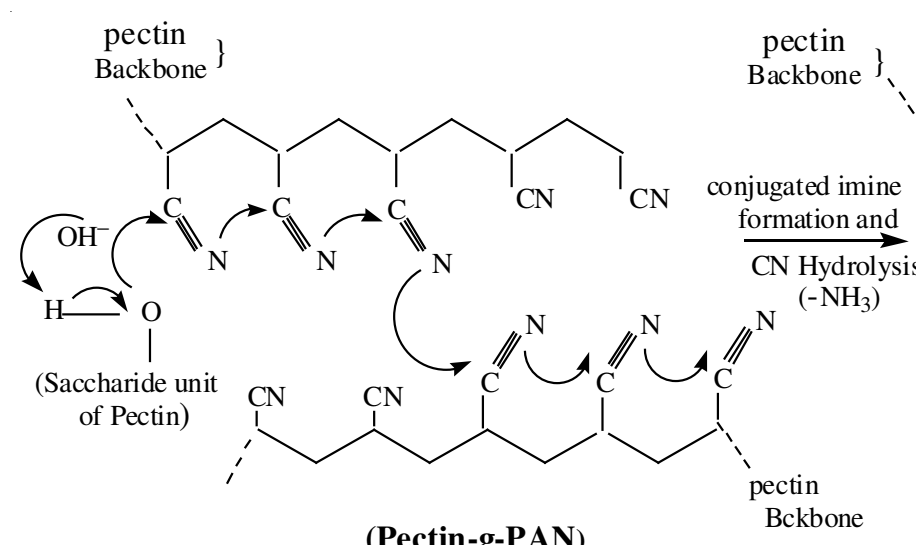

(Pectin-g-PAN)

(white-cream color)

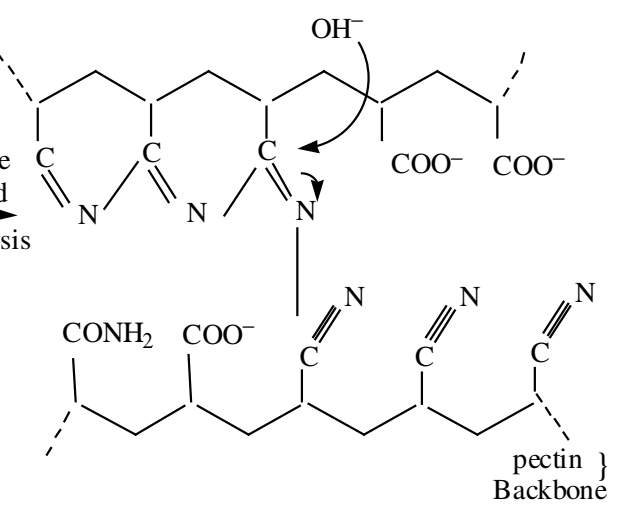

(deep red)

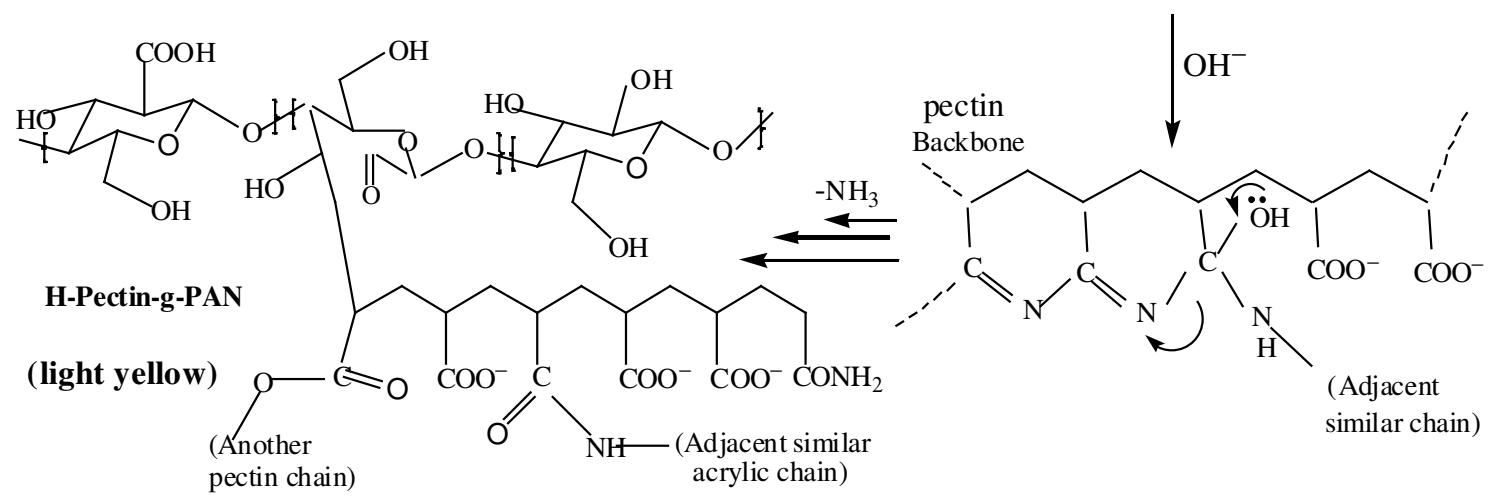

Scheme-I: Proposed mechanism for crosslinking during the hydrolysis of nitrile groups of the Pec-PAN mixture to produce the Pec-g-poly(AN) hydrogel

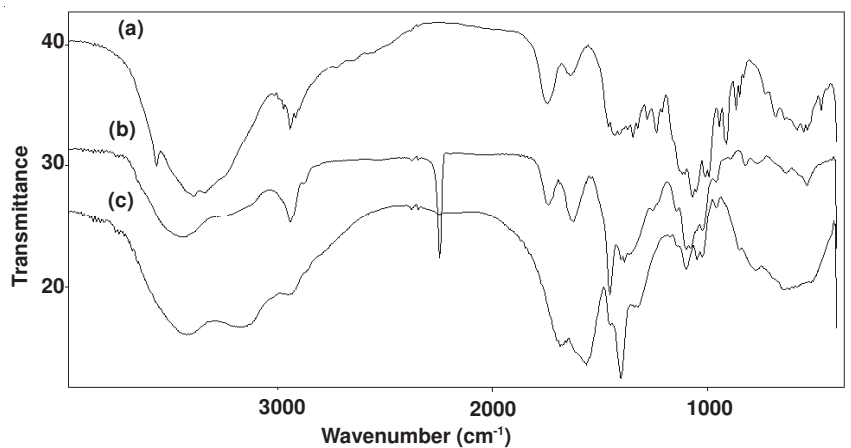

Fig. 2. FTIR spectra of pectin (a), pectin- $g$-PAN pec-poly(NaAA-co-AAm) (b) and (c) H-pectin- $g$-PAN

band of the pectin portion of the copolymer. As shown in Fig. $2 \mathrm{c}$ and Scheme-I, after alkaline hydrolysis, most of the nitrile groups are converted to carboxamide and carboxylate groups.

To obtain an additional evidence of in situ crosslinking during alkaline hydrolysis, a similar reaction was conducted in absence of the polysaccharide. Since the resulted product became soluble, the crosslinks really formed between the alkoxide ions of Pectin and the nitrile groups of polyacrylonitrile. This fact practically proves that the starch hydroxyls are involved in the crosslinking.

Standard calibration curve: The calibration curve of the absorbance as a function of the ephedrine concentration at $276 \mathrm{~nm}$, shown in Fig. 3, has a linear relationship with a correlation coefficient (r) of 0.997 and 0.996 at pHs 1.6 and 7.4, respectively.
$\mathrm{pH}=1.6$
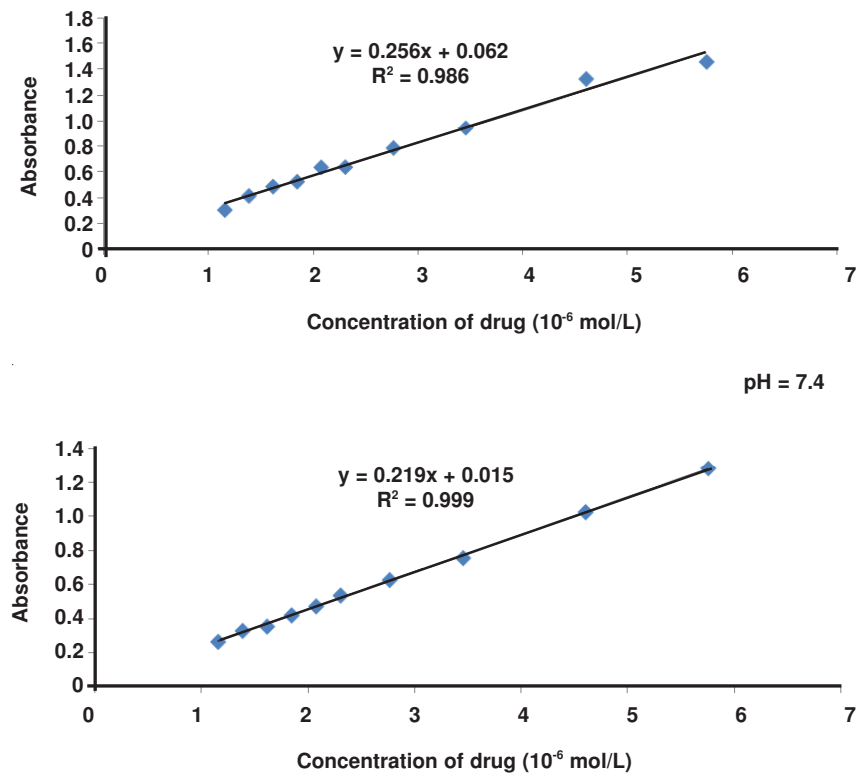

Fig. 3. Standard calibration curve of the absorbance as a function of ephedrine concentration at $276 \mathrm{~nm}$ on the UV spectrophotometer at $\mathrm{pH} 1.6$ (a) and $\mathrm{pH} 7.4$ (b)

Ephedrine loading: For the investigation of drug adsorption behaviour of hydrogels prepared in this study, hydrogels were firstly swollen in ephedrine solution in concentration range $0.20-2.80 \mathrm{mg} / \mathrm{mL}$. As can be seen from the Fig. 4, an 


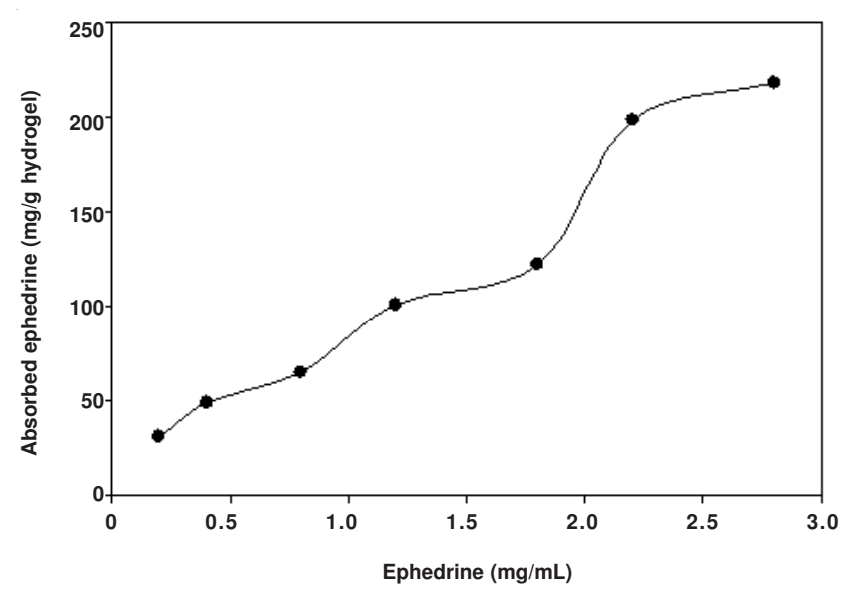

Fig. 4. Effect of drug concentration on the adsorption capacities of hydrogel

increase in drug concentration in the swelling medium increased the amount of adsorbed drug, as observed in many adsorption studies ${ }^{13-15}$. This result can be also obtained from the following equation:

$$
\mathrm{q}_{\mathrm{e}}=\left(\frac{\left(\mathrm{C}_{\mathrm{i}}-\mathrm{C}\right)}{\mathrm{m}}\right) \times \mathrm{V}_{\mathrm{t}}
$$

where $\mathrm{q}_{\mathrm{e}}$ is in $\mathrm{mg}$ adsorbate per gram of dry adsorbent, $\mathrm{C}_{\mathrm{i}}$ and $\mathrm{C}$ are the initial and equilibrium concentrations of adsorbate solution in $\mathrm{mg} / \mathrm{mL}, \mathrm{V}_{\mathrm{t}}$ is the volume of solution treated in $\mathrm{mL}$ and $\mathrm{m}$ is the mass $(\mathrm{g})$ of dry adsorbent. As can be seen from eqn. 3 , an increase in concentration of drug in the gel system increased $\mathrm{q}_{\mathrm{e}}$ values.

The amounts of the loaded drug in superabsorbent hydrogels was also significantly affected by the impregnation times (Fig. 5). It is obvious that with increasing the loading time, the amount of drug loaded is initially increased and then begins to level off. The initial increment in the amounts of the loaded drug with increasing the loading time can be ascribed to the increased drug diffusion into the swollen matrix. The most efficient time of loading efficiency was $18 \mathrm{~h}$, where a major amount of drug was encapsulated ${ }^{16}$.

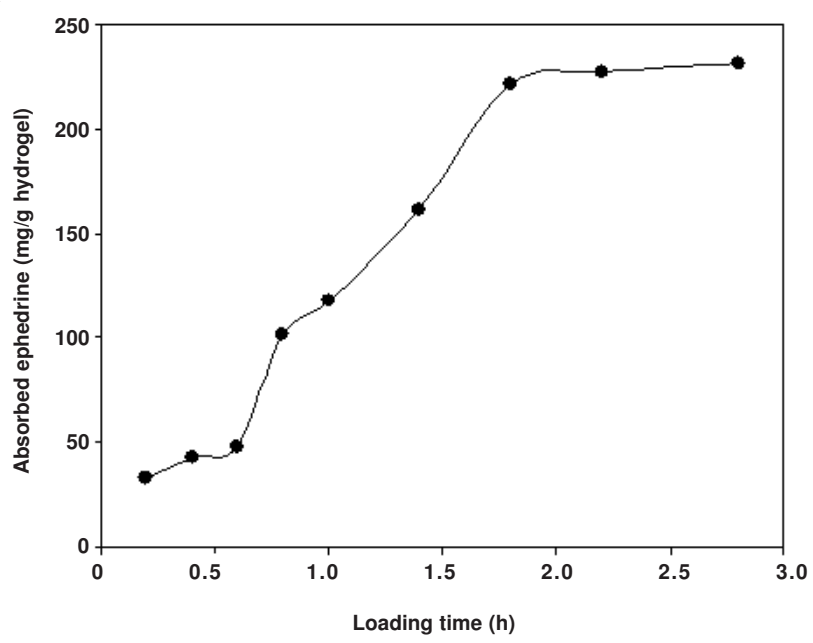

Fig. 5. Dependency of the drug loading amount to the loading time (h)

In vitro release behaviour of hydrogels: To determine the potential application of pectin-based superabsorbent containing a pharmaceutically active compound, we have investigated the drug release behaviour form this system under physiological conditions. The per cent of released drug from the polymeric carriers as a function of time is shown in Fig. 6. The concentration of ephedrine released at selected time intervals was determined by UV spectrophotometer. The drugloaded hydrogels with high degrees of drug loading (> $85 \%$ ) were prepared by the swelling-diffusion method. The amount of ephedrine released in a specified time from the pectin-based hydrogel decreased as the $\mathrm{pH}$ of the dissolution medium was lowered (Fig. 7). At low pH values, electrostatic repulsion between the carboxylic acid groups of backbone is low, thus decreases gel swelling and minimizes release of ephedrine via diffusion. However, in alkaline media the presence of $\mathrm{OH}-$ increases the electrostatic repulsion between carboxylic acid groups, thus increases the gels swelling degree and so the release of ephedrine increased ${ }^{17-19}$.

(a)

$\mathrm{pH}=1.6$

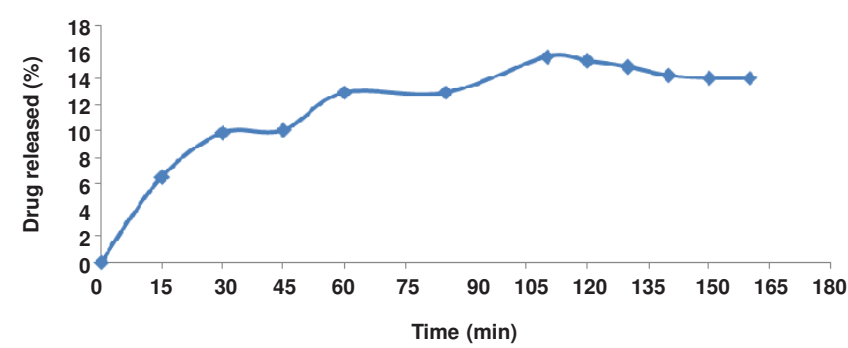

(b)

$\mathrm{pH}=7.4$

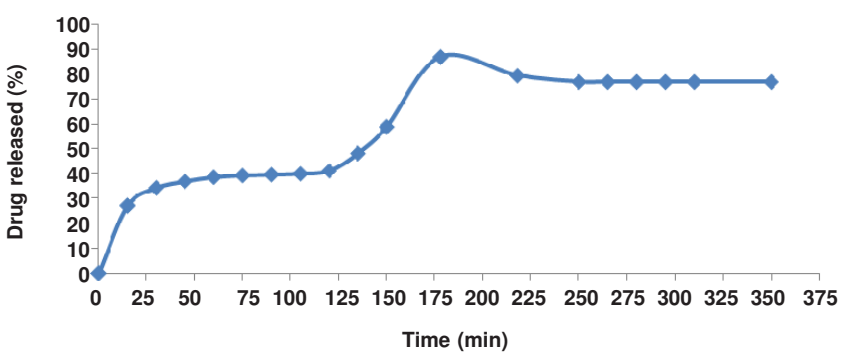

Fig. 6. Dependency of the per cent releasing drug to the time in $\mathrm{pH}=1.6$ (a) and $\mathrm{pH}=7.6$ (b)

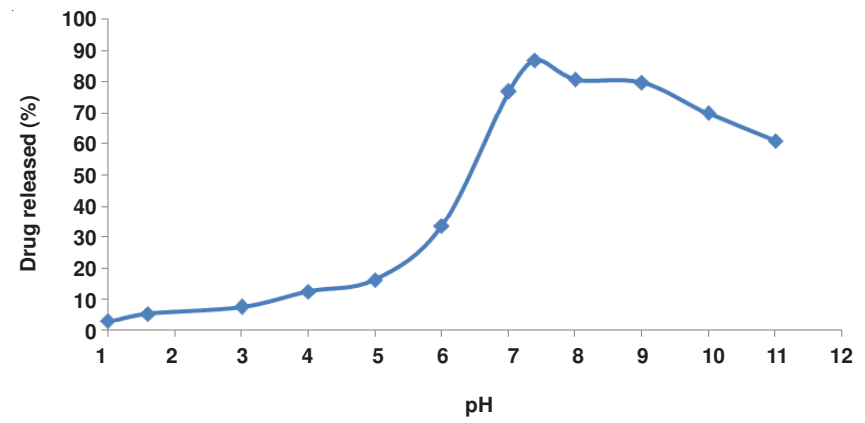

Fig. 7. Release of ephedrine from hydrogel carrier as a function of time and $\mathrm{pH}$ at $37^{\circ} \mathrm{C}$

\section{Conclusion}

The superabsorbent hydrogel, Pec-poly(AN), was synthesized through alkaline hydrolysis of pectin-PAN physical mixture. The reaction of pectin alkoxide anions with nitrile groups of polyacrylonitrile, forms crosslinking points and 
results in a three-dimensional network. Because a polymerization reaction is not involved, so there is no need to initiator, toxic and/or expensive monomer and crosslinker. Therefore, problems such as polymerization control, conversion loss and residual monomer are eliminated. Indeed, since no toxics material is used for the synthesis, this practical approach may be preferred to as a relatively "green process". The superabsorbent hydrogels exhibited high sensitivity to $\mathrm{pH}$, so that, several swelling changes of the hydrogel were observed in $\mathrm{pH}$ variations of a wide range (1-13). Ionic repulsion between charged groups incorporated in the gel matrix by an external $\mathrm{pH}$ modulation could be assumed as the main driving force responsible for such abrupt swelling changes. Furthermore, the reversible swelling-deswelling behaviour in solutions with acidic and basic $\mathrm{pH}$ makes the hydrogels a suitable candidate for controlled drug delivery systems.so, the loading and release of ephedrine from the $\mathrm{pH}$-sensitive hydrogels was effective. The release value of ephedrine from hydrogels at $\mathrm{pH} 7.4$ was higher than that at $\mathrm{pH} 1.6$ due to the electrostatic repulsion between carboxylate groups. The hydrogels presented in this study may serve as a platform for a wide range of pharmaceutical uses to improve the bioavailability of non-steroidal antiinflammatory drugs.

\section{REFERENCES}

1. F.L. Buchholz and A.T. Graham, In: Modern Superabsorbent Polymer Technology, Wiley, New York (1997).

2. H. Cheng, J.L. Zhu, Y.X. Sun, S.X. Cheng, X.Z. Zhang and R.X. Zhuo, Bioconjug. Chem., 19, 1368 (2008).

3. L.Y. Chu, J.W. Kim, R.K. Shah and D.A. Weitz, Adv. Funct. Mater., 17, 3499 (2007).

4. L.Y. Chu, T. Yamaguchi and S. Nakao, Adv. Mater., 14, 386 (2002).

5. V. Crescenzi, L. Cornelio, C. Di Meo, S. Nardecchia and R. Lamanna, Biomacromolecules, 8, 1844 (2007).

6. D.T. Eddington and D.J. Beebe, Adv. Drug Deliv. Rev., 56, 199 (2004).

7. M. Hamidi, A. Azadi and P. Raflei, Adv. Drug Deliv. Rev., 60, 1638 (2008).

8. H. Koo, G. Jin, H. Kang, Y. Lee, H.Y. Nam, H. Jang and G.S. Park, Int. J. Pharm., 374, 58 (2009).

9. H. Kranz and R. Bodmeier, Eur. J. Pharm. Sci., 34, 164 (2008).

10. I.C. Kwon, Y.H. Bae and S.W. Kim, Nature, 354, 291 (1991).

11. J.K. Oh, R. Drumright, D.J. Siegwart and K. Matyjaszewski, Prog. Polym. Sci., 33, 448 (2008).

12. J. Siepmann and N.A. Peppas, Adv. Drug Deliv. Rev., 48, 139 (2001).

13. K.S. Soppimath, T.M. Aminabhavi, A.M. Dave, S.G. Kumbar and W.E. Rudzinski, Drug Dev. Ind. Pharm., 28, 957 (2002).

14. T. Tatsuma, K. Takada and T. Miyazaki, Adv. Mater., 19, 1249 (2007).

15. P.D. Thornton, R.J. Mart and R.V. Ulijn, Adv. Mater., 19, 1252 (2007).

16. W. Wang, L. Liu, X.J. Ju, D. Zerrouki, R. Xie, L. Yang and L.Y. Chu, Chem. Phys. Chem., 10, 2405 (2009).

17. M. Yang, L.Y. Chu, H.D. Wang, R. Xie, H. Song and C.H. Niu, Adv. Funct. Mater., 18, 652 (2008).

18. M. Sadeghi and M. Yarahmadi, Orient. J. Chem., 27, 417 (2011).

19. M. Sadeghi and M. Yarahmadi, J. Chem., 27, 453 (2011). 